\title{
Ladrillos Cerámicos Porosos a partir de Mezclas de Arcilla y Carozos de Durazno
}

\section{Porous ceramic bricks from clay and peach pits mixtures}

Presentación: 06/02/2020

Aprobación: 29/04/2020

\author{
Nancy Quaranta \\ Grupo de Estudios Ambientales - Facultad Regional San Nicolás - Universidad Tecnológica Nacional - \\ Argentina \\ Investigador Comisión de Investigaciones Científicas. \\ nquaranta@frsn.utn.edu.ar.
}

\section{Miguel Unsen}

Grupo de Estudios Ambientales - Facultad Regional San Nicolás - Universidad Tecnológica Nacional Argentina

munsen@frsn.utn.edu.ar

\section{Hugo López}

Grupo de Estudios Ambientales - Facultad Regional San Nicolás - Universidad Tecnológica Nacional Argentina

halopez@frsn.utn.edu.ar

\section{Adrián Cristóbal}

Instituto de Investigaciones en Ciencia y Tecnología de Materiales - Universidad Nacional de Mar del Plata - Consejo Nacional de Investigaciones Científicas y Técnicas - Argentina acristobal@fi.mdp.edu.ar

\section{Resumen}

El objetivo de este trabajo es analizar la factibilidad de uso de carozos de durazno, desecho biomásico del sector frutihortícola, como formador de poros en la fabricación de piezas cerámicas alivianadas. Se prepararon mezclas de arcilla comercial con el agregado de 5\%, $10 \%, 15 \%$ y $20 \%$ en volumen de carozos y una muestra de arcilla sin agregados a modo de referencia. Todas las mezclas con un agregado de $8 \%$ de humedad se conformaron en moldes de $70 \mathrm{~mm}$ x $40 \mathrm{~mm}$, utilizando una presión de compactación de $25 \mathrm{MPa}$. El tratamiento térmico fue $1000^{\circ} \mathrm{C}$ durante 3 horas, a una velocidad de calentamiento de $1^{\circ} \mathrm{C} / \mathrm{min}$. Los resultados obtenidos reflejan una alta factibilidad de utilización de esta biomasa para el uso 
estudiado. Los productos cerámicos obtenidos poseen buenas propiedades generales, aunque las propiedades mecánicas son un limitante ya que cumplen con los requerimientos del mercado hasta agregados del 10\% de material residual.

Palabras claves: Biomasa, Residuos, Cerámicos.

\begin{abstract}
The objective of this work is to analyze the feasibility of using peach pits, biomass waste from the fruit and vegetable sector, as a pore former in the manufacture of lightweight ceramic pieces. Commercial clay mixtures were prepared with the aggregate of 5\%, 10\%, 15\% and $20 \%$ by volume of pits and a clay sample without aggregates for reference. All mixtures with an aggregate of $8 \%$ humidity were formed in molds of $70 \mathrm{~mm} \times 40 \mathrm{~mm}$, using a compaction pressure of $25 \mathrm{MPa}$. The heat treatment was $1000^{\circ} \mathrm{C}$ for 3 hours, at a heating rate of $1^{\circ} \mathrm{C} /$ min. The obtained results reflect a high feasibility of using this biomass for the studied use. The ceramic products have good general properties, although the mechanical properties are a limitation since they meet the market requirements up to $10 \%$ aggregates of residual material.
\end{abstract}

Keywords: Biomass, Wastes, Ceramics.

\title{
Introducción
}

Dentro de las clasificaciones de residuos, el término residuo de biomasa se refiere a la materia orgánica originada por un proceso natural o inducido que puede utilizarse como fuente de energía, esto es, aquel material residual que puede ser utilizado como combustible.

Numerosas biomasas residuales han sido estudiadas en diversos usos, incluyendo procesos biomasa-energía, como adsorbentes de metales pesados contenidos en efluentes líquidos, como fuentes de sustancias químicas de mayor valor añadido, como materias primas en la industria de la construcción, etc. (Quaranta et al, 2017; Saidur et al, 2011).

Se han propuesto y se han aplicado numerosos cambios en los procesos productivos con las denominadas tecnologías limpias y de minimización, que tienden por un lado a reducir en origen la generación de residuos, y por el otro a reutilizarlos transformándolos en subproductos de otras industrias. La valorización de éstos es precisamente la clave para lograr que se dé un vuelco a esta situación interesando al sector industrial guiado por motivos de productividad, y al sector científico en su espíritu investigador.

En la literatura se hallan estudios de factibilidad de reutilización de una gran diversidad de estos residuos industriales considerados residuos de biomasa. En particular, en lo que respecta a residuos de la industria alimenticia se han encontrado numerosos trabajos fundamentalmente referidos a su utilización como combustibles, denominados procesos biomasa-energía (Rabaçal et al, 2013; De Velasco Maldonado et al, 2016; Mao et al, 2018; Bonja et al, 2019; Nunez et al, 2020; da Silva et al, 2020).

Los recursos biomásicos se encuentran disponibles en distintas formas, incluyendo cultivos energéticos dedicados, subproductos de agricultura, subproductos forestales, plantas acuáticas, subproductos de origen municipal, subproductos animales y hortícolas, subproductos de la industria de la alimentación, entre otros (Damien, 2010). Las empresas procesadoras de frutas desechan grandes cantidades de materia orgánica que no es aprovechada, 
debido a que las empresas dirigen su mayor esfuerzo hacia el aprovechamiento de la pulpa para la realización de productos alimenticios de mayor valor agregado.

El material que se estudió en esta investigación es el carozo de durazno. Esta biomasa ha sido investigada principalmente para la producción de carbón activado para diferentes usos. Martín Martínez et al (2018) ha investigado la actividad de carbones sintetizados a partir de carozos de durazno para su uso como catalizador en la oxidación de peróxidos.

Por su parte, Tsoncheva et al (2018) han utilizado carbones activados a partir de esta biomasa como soporte de catalizadores en la descomposición de metanol. La aplicación de estos carbones activados como sensores de detección de cafeína ha sido analizada por Arroyo Gómez et al (2018). También se ha estudiado el uso de carbón activado a partir de esta biomasa como adsorbente de contaminantes (Alvarez Torrellas et al, 2015).

Los carozos de durazno han sido analizados además como fuente de derivados químicos para la obtención de péptidos (Vázquez Villanueva et al, 2015).

En este trabajo, este residuo primario de biomasa se analizó para su valorización como agregado en la fabricación de cerámicos. Esta materia prima al ser incorporada a la matriz arcillosa, se constituye como material formador de poros, ya que a las temperaturas de trabajo combustionan dentro del ladrillo produciendo gases y constituyentes inorgánicos (cenizas), dando como resultado esperado lo que se denomina "ladrillo alivianado". Este tipo de biomasa residual se produce en grandes cantidades en las áreas frutícolas de Argentina, en particular en la zona de Cuyo, y es obtenido como material residual del propio proceso productivo.

En la industria cerámica, la porosidad de los materiales producidos es manejada normalmente hacia menores o mayores valores, según el requerimiento del producto y su aplicabilidad. En el caso de aumento de la porosidad, suelen utilizarse compuestos orgánicos, derivados de la industria del petróleo, como por ejemplo partículas o espumas de polietileno o poliuretano (Ferrer et al, 2011).

El objetivo de este trabajo es analizar la factibilidad de utilización de un residuo biomásico como los carozos de durazno, como agregados a matrices arcillosas, con el fin de incrementar la porosidad de las piezas cerámicas obtenidas, manteniendo las propiedades de este tipo de productos dentro de los requerimientos del mercado. Tanto las materias primas (carozos y arcilla) como los productos obtenidos fueron analizados por las técnicas habituales para este tipo de estudios.

\section{Desarrollo}

Para el desarrollo de este trabajo se utilizan como materias primas, carozos de durazno enteros, provenientes de una empresa de la zona frutihortícola de Cuyo y arcilla comercial en forma granular, obtenida de una empresa cerámica de la ciudad de Rosario.

Estos materiales se caracterizaron por diversas técnicas: microscopía electrónica de barrido (SEM), análisis dispersivo de energía de rayos X (EDS), difracción de rayos X (XRD), distribución granulométrica, análisis térmico diferencial y termogravimétrico (DTA-TGA), pérdida de peso por calcinación (PPC), ecotoxicidad, entre otras.

Los análisis SEM se llevaron a cabo con un microscopio marca Philips 515 anexado a un EDS (EDAX-Phoenix) que se utilizó para el análisis químico. Los patrones de difracción de rayos $\mathrm{X}$ de los polvos se obtuvieron con un equipo PANalytical X'Pert PRO, con radiación $\operatorname{CuKa}(\lambda=1,5406 \mathrm{~nm})$. 
Para los ensayos de DTA-TGA se utilizó un equipo Shimadzu DTA-50, TGA-50 con analizador TA-50 WSI, utilizando velocidades de calentamiento de $1^{\circ} \mathrm{C} / \mathrm{min}$, en el rango de temperaturas ambiente $-1100^{\circ} \mathrm{C}$, en atmósfera de aire.

Para el ensayo granulométrico se utilizó un equipo mecánico marca Zonytest, y un conjunto de tamices de mallas de acero inoxidable certificados según normas ASTM.

Los ensayos de ecotoxicidad se realizaron mediante una adaptación de la Norma IRAM 29114:2008, utilizando semillas de ray-grass, como especie sensible. Este ensayo se realiza debido a que los materiales residuales, al ser depositados sobre el suelo, para disposición o almacenamiento, pueden afectar las características del suelo que los contienen. Para realizar este ensayo, se obtienen elutriados, que luego se diluyen a diferentes concentraciones, y se analiza el efecto que ellos tienen sobre el crecimiento de la radícula de la especie sensible. Se realizan además muestras de referencia con agua destilada por un lado, y en contacto directo con el residuo por el otro. Luego de cinco días a $25^{\circ} \mathrm{C}$, se determina la inhibición en el crecimiento de la radícula de los especímenes estudiados (\%IR).

Tanto la arcilla como el residuo fueron previamente secados a $100^{\circ} \mathrm{C}$, y luego molidos y tamizados. Los carozos de durazno cuando salen de la industria procesadora contienen parte de la pulpa húmeda adherida, por ello es necesario secarlos antes de molerlos. El carozo completo (semilla y endocarpio) es molido hasta obtener material en polvo para las caracterizaciones. En la Figura 1 se muestran imágenes representativas de estas etapas.

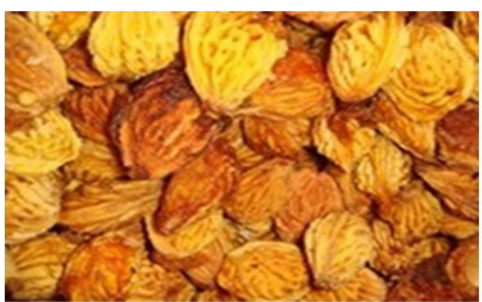

(a)

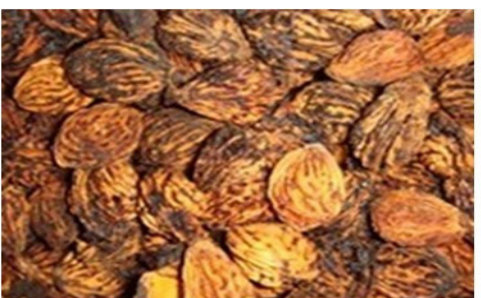

(b)

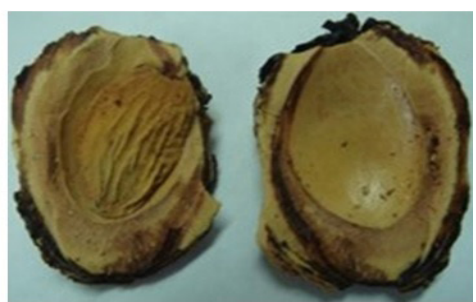

(c)

Figura 1. Tratamiento de los carozos. (a) Carozos con pulpa adherida. (b) Carozos secos. (c) Carozo listo para moler (semilla y endocarpio).

Ambos materiales se utilizaron con un tamaño de partícula inferior a $1 \mathrm{~mm}$, tamaño apropiado para la incorporación en mezclas con arcilla. Se prepararon mezclas de arcilla comercial con el agregado de 5\%, 10\%, 15\% y 20\% en volumen de carozos de durazno y una muestra de arcilla sin agregados a modo de referencia. El peso de arcilla en cada muestra fue de $80 \mathrm{~g}$, a los efectos de lograr, luego del prensado, un tamaño proporcional al ladrillo tradicional.

Todas las mezclas con un agregado de $8 \%$ de humedad se conformaron en moldes de 70 $\mathrm{mm}$ x $40 \mathrm{~mm}$, resultando espesores entre $15 \mathrm{~mm}$ y $18 \mathrm{~mm}$ aproximadamente, utilizando una presión de compactación de $25 \mathrm{MPa}$. Estas probetas fueron sometidas a un tratamiento térmico de $1000^{\circ} \mathrm{C}$ durante 3 horas, con una velocidad de calentamiento $1^{\circ} \mathrm{C} / \mathrm{min}$, siguiendo una rampa de calentamiento de 16 horas y una de enfriamiento de 18 horas, aproximadamente.

Para el tratamiento térmico se utilizó un horno eléctrico TecnoDalvo con controlador de temperatura programable Dhacel TD101.

Los productos cocidos obtenidos se caracterizaron por las técnicas habituales para materiales cerámicos, que conducen a determinar las propiedades fisicoquímicas, morfológicas, 
microestructurales y mecánicas, que permiten inferir sus aptitudes en servicio. Entre dichas técnicas pueden mencionarse: microscopía óptica y electrónica de barrido, porosidad, pérdida de peso por calcinación, variación volumétrica permanente y resistencia a la flexión.

Las observaciones por microscopía óptica se realizaron con un equipo Zeiss-Axiotech con una cámara Donpisha 3CCD y procesador de imágenes.

La porosidad de las muestras se determinó siguiendo la norma IRAM 12510.

El ensayo mecánico se llevó a cabo con una máquina de ensayos universal Cific de $294 \mathrm{kN}$.

Las muestras cerámicas producidas serán identificadas en adelante de la siguiente manera:

CD0: muestra de referencia de arcilla sin agregado de biomasa.

CD5: muestra con 5\% en volumen de carozos molidos.

CD10: muestra con 10\% en volumen de carozos molidos.

CD15: muestra con 15\% en volumen de carozos molidos.

CD20: muestra con $20 \%$ en volumen de carozos molidos.

Resultados y discusión

La distribución granulométrica de los materiales se presenta en la Figura 2. En el caso del residuo se observa que aproximadamente el 95\% de las partículas presenta tamaños entre 125 y $500 \mu \mathrm{m}$. La arcilla, además de tener una distribución de tamaños más homogénea, presenta una mayor cantidad de partículas más finas, favoreciendo la integración de las mezclas respecto a la relación de tamaños de partículas normalmente buscada.

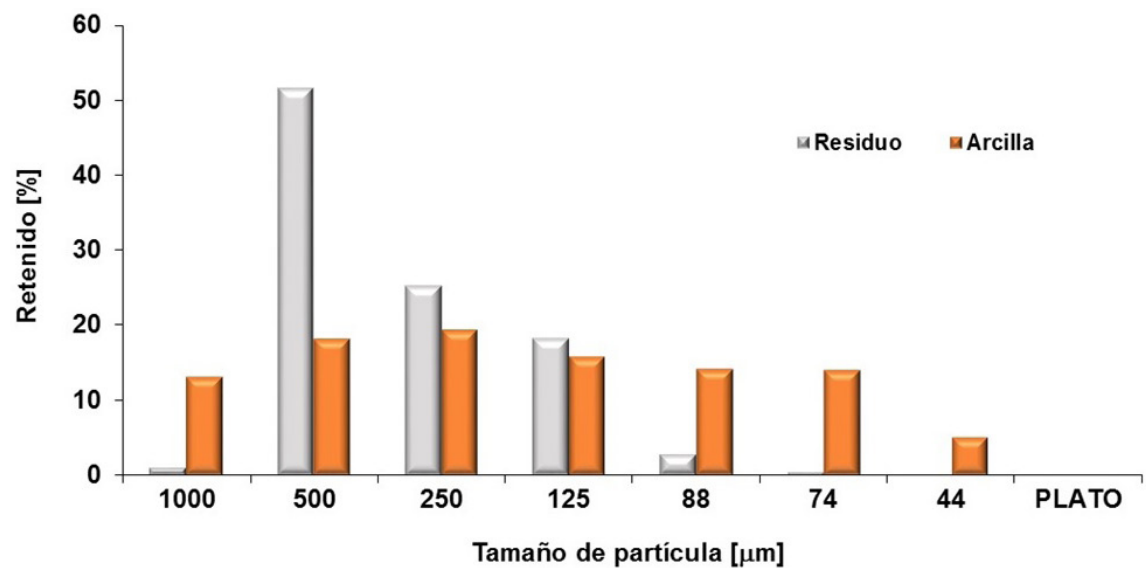

Figura 2: Distribución granulométrica del residuo y arcilla.

La Figura 3 muestra la apariencia microscópica de los carozos por SEM. Se observa una estructura fibrosa en la superficie exterior y granular en el interior. El análisis químico semicuantitativo por EDS muestra, además de grandes cantidades de $\mathrm{C}$ y $\mathrm{O}$, la presencia de elementos como Na, K, Mg, Al, Si y Fe. 

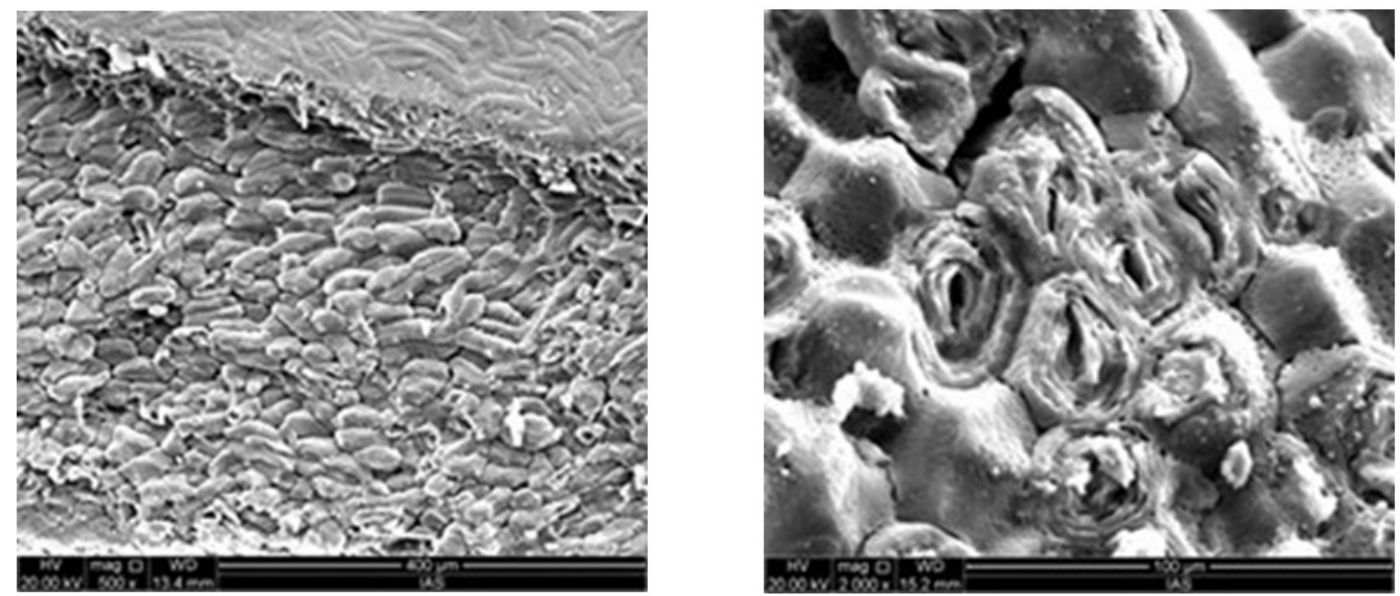

Figura 3: Imágenes SEM de los carozos de durazno.

Los resultados de DTA-TGA de la muestra de carozos en polvo, se presentan en la Figura 4. Se observan algunos picos exotérmicos que pueden ser asignados a la combustión de los diversos biopolímeros que conforman la estructura, como hemicelulosa (HC), celulosa (C) y lignina (L).

A partir de estas curvas, es posible estimar la composición de este material en relación con el contenido de gases y agua adsorbidos, biopolímeros y fracción inorgánica (formación de cenizas). A medida que la estructura de los biopolímeros (hemicelulosa, celulosa y lignina) se vuelve más compleja, las temperaturas de combustión son más altas.

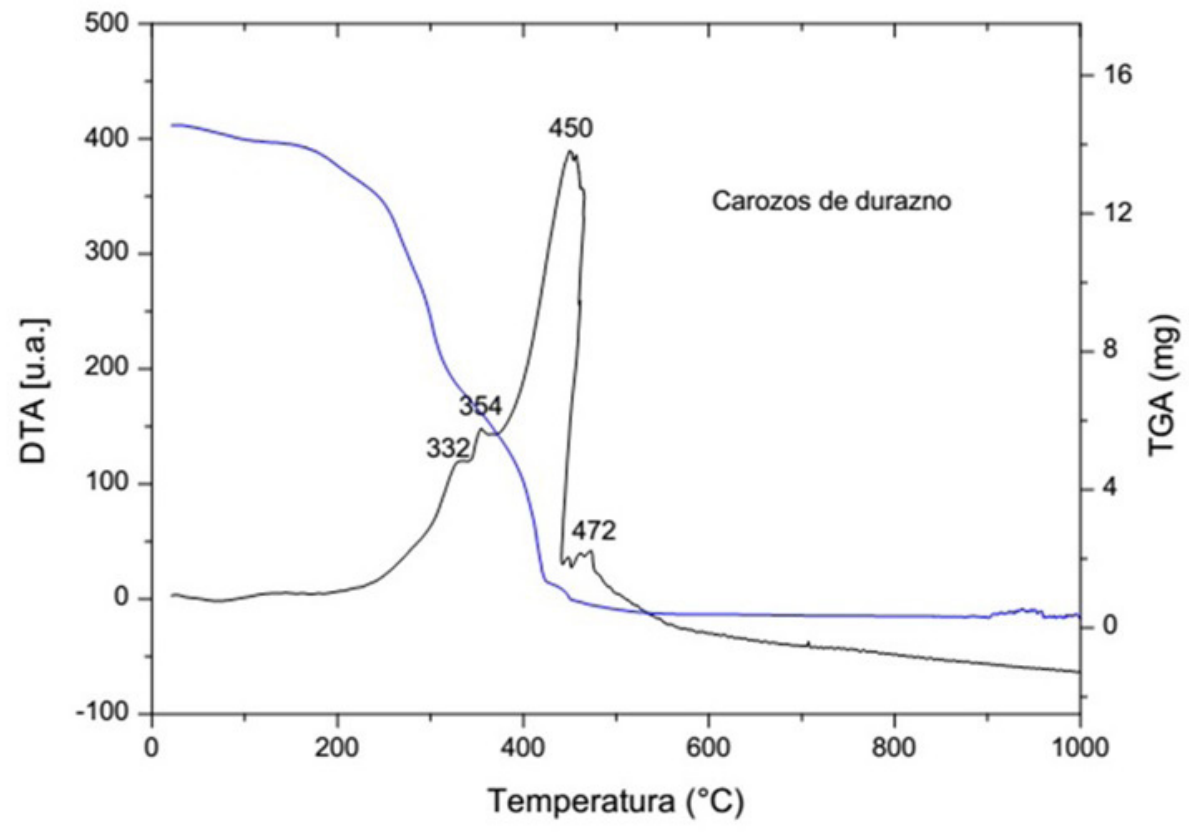

Figura 4. DTA-TGA del residuo carozo de durazno 


\begin{tabular}{|c|c|}
\hline Compuesto & Composición \% \\
\hline H2O + gases & 4,9 \\
\hline HC - C & 83,1 \\
\hline L & 9,6 \\
\hline cenizas & 2,4 \\
\hline
\end{tabular}

Tabla 1. Composición estimada del residuo carozo de durazno a partir de DTA-TGA.

El análisis DTA-TGA muestra que este material orgánico se quema en un amplio rango de temperatura, entre $300^{\circ} \mathrm{C}$ y $550^{\circ} \mathrm{C}$. Esto es importante para garantizar que cuando este material se incorpore en mezclas de arcilla como formador de poros, el proceso de sinterización se realice sin formación de grietas en el ladrillo. Estos resultados además indican que después del tratamiento térmico, el material residual que eventualmente se incorporará a los ladrillos (cenizas) es inferior al 3\%. La Tabla 1 muestra la composición estimada a partir de las curvas de la Figura 4.

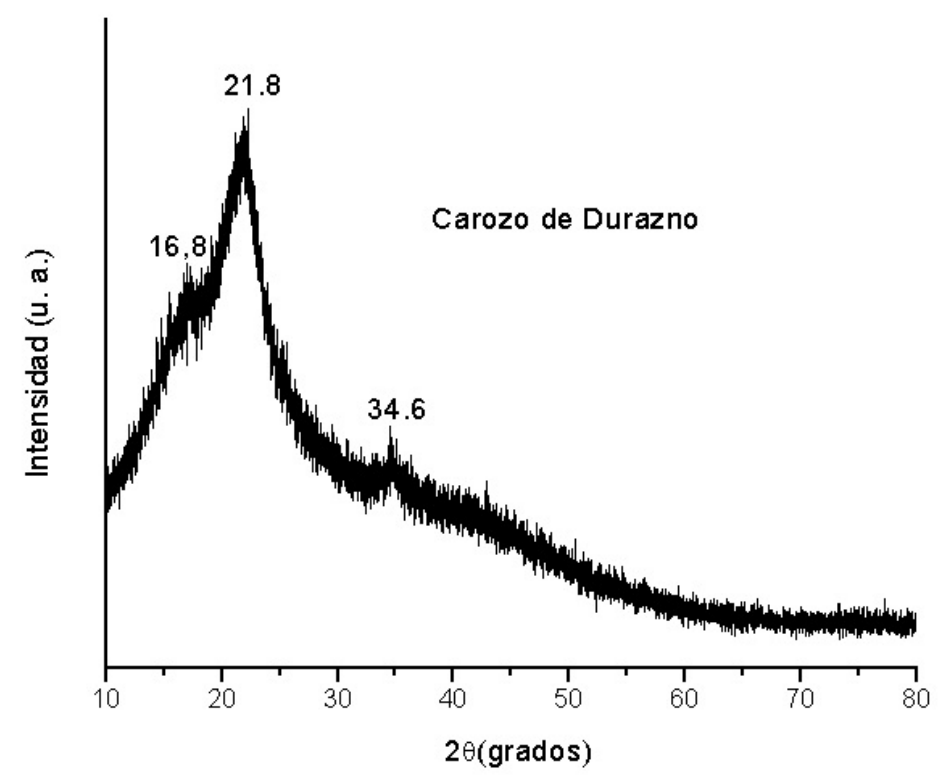

Figura 5. XRD de la biomasa residual

El análisis XRD de los carozos en polvo, muestran la presencia de algunos picos correspondientes a la celulosa semicristalina, como puede observarse en la Figura 5.

Los resultados del ensayo de ecotoxicidad realizado para el residuo biomásico en polvo, se muestran en la Figura 6, expresado como porcentaje de inhibición del crecimiento de 
la radícula [\%IR], en función de las concentraciones del elutriado y sus diluciones, y de las muestras de referencia (agua destilada y residuo). Como se determina a partir de la figura, este residuo resulta ecotóxico aún en bajas cantidades, por lo que no puede ser dispuesto sin control, ni almacenado en sitios que no sean acondicionados para ello.

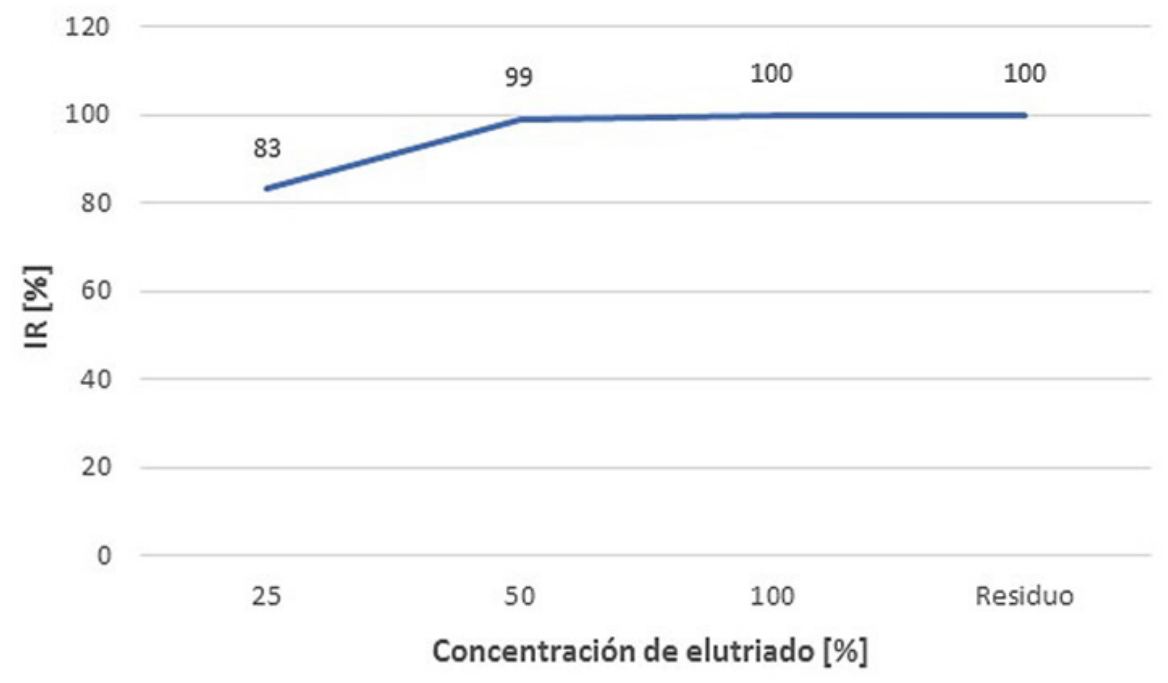

Figura 6. Análisis de ecotoxicidad de los carozos de durazno

Las muestras cocidas presentan en todos los casos una coloración rojiza debido a los contenidos de hierro de la arcilla. Presentan muy buenas características macroscópicas, con mantenimiento de aristas y sin desgranamiento de la estructura, lo que indica que se ha logrado un grado de sinterización adecuado. Estas piezas obtenidas se presentan en la Figura 7, identificadas como CD0, CD5, CD10, CD15 y CD20.
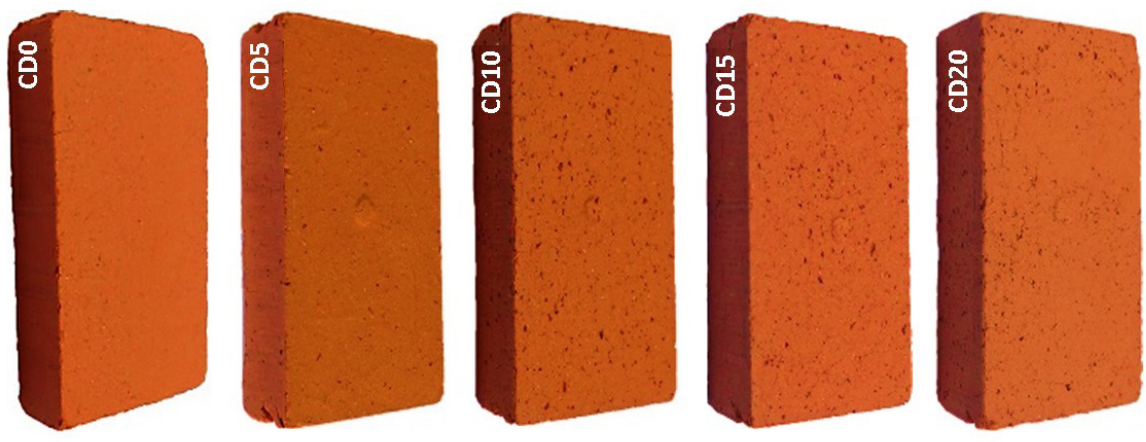

Figura 7: Ladrillos cerámicos cocidos

La porosidad determinada de las probetas se presenta en la Figura 8. Se observa que a medida que aumenta el contenido de residuo, la porosidad de las piezas también aumenta. Este resultado coincide con la observación directa de manera macroscópica de las piezas 
obtenidas, como se aprecia en la figura anterior. La porosidad de los ladrillos ha sido analizada también por microscopía óptica. La Figura 9 presenta imágenes tomadas en el interior de los ladrillos, donde resulta apreciable como varía la cantidad y tamaños de los poros presentes en las muestras que han sido obtenidas con agregados de carozos (CD10 y CD20), en comparación con el ladrillo producido utilizando solamente arcilla comercial (CD0).

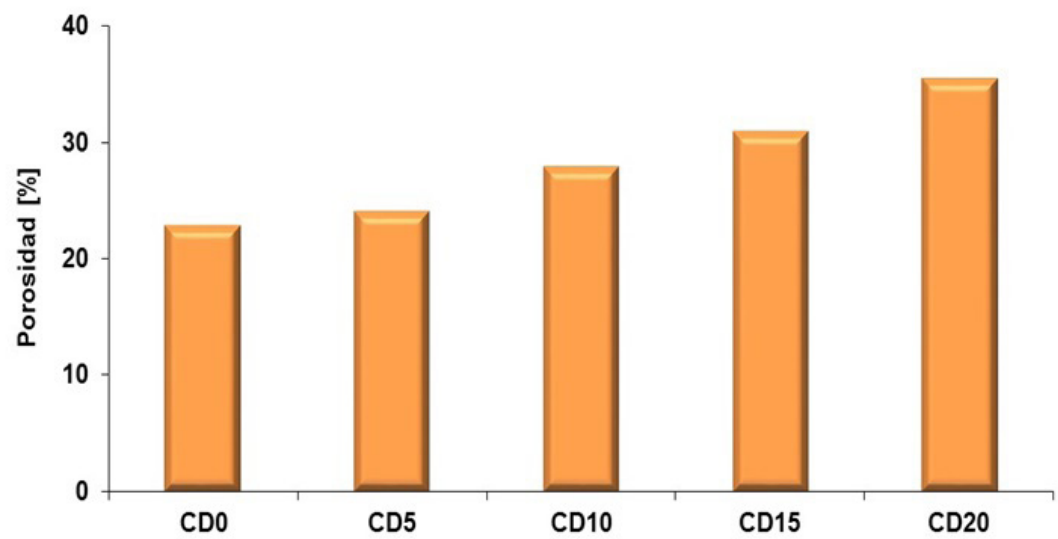

Figura 8: Porosidad de los compactos.
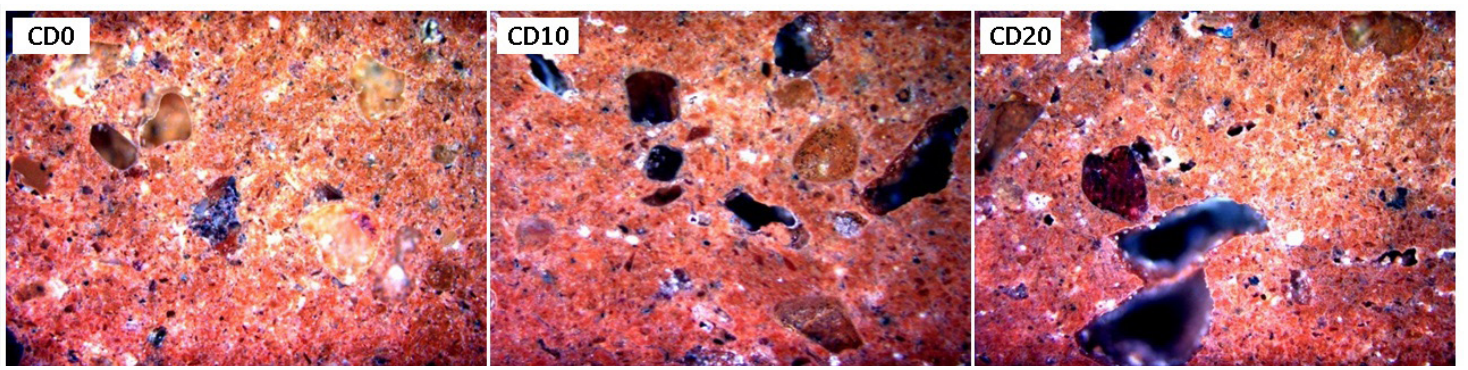

Figura 9: Imágenes por microscopía óptica del interior de los ladrillos. Aumento: 50X.

La pérdida de peso por calcinación (PPC) de las muestras se presenta en la Figura 10. Se puede apreciar que como es de esperar la mayor pérdida de peso es registrada para las muestras con mayor contenido de residuo, siendo el máximo valor del 18\% aproximadamente. Las PPC determinadas corresponden a la pérdida correspondiente a la arcilla (material orgánico, compuestos volátiles, o compuestos que se descomponen), al agua agregada que se evapora, y además a la pérdida por combustión de la biomasa (carozos) en el caso de las muestras con descarte agregado, que combustiona casi en su totalidad. La cantidad de ceniza originada en este último proceso es muy baja $(<3 \%)$, y la misma quedará integrada a la estructura del ladrillo. 


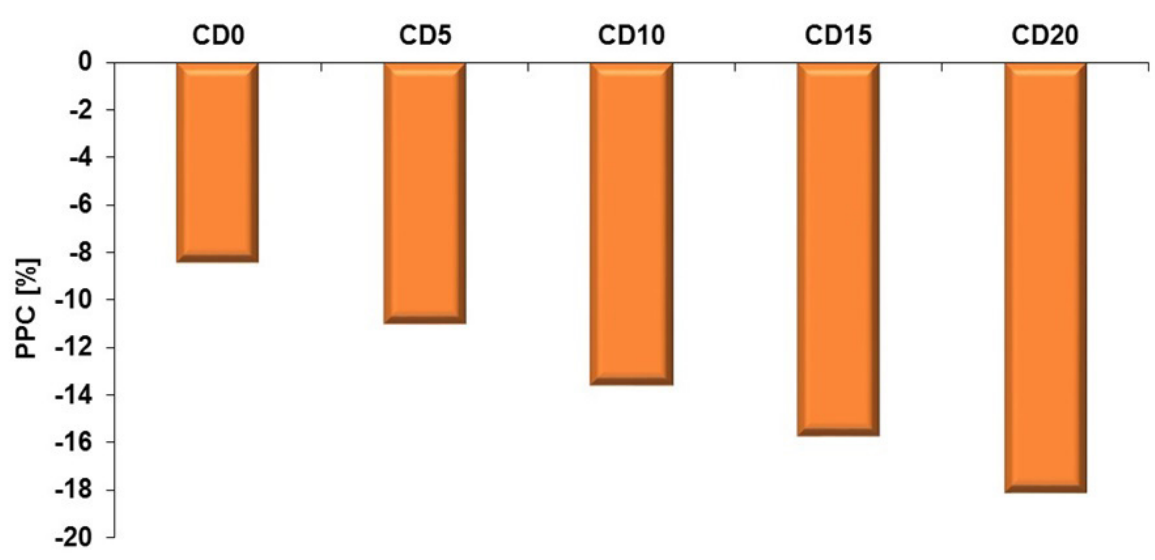

Figura 10: Pérdida de peso por calcinación de los ladrillos (PPC).

La resistencia a la flexión (o módulo de rotura-MOR) de los compactos se presenta en la Figura 11, donde se observa que a medida que aumenta el contenido de descarte, disminuye la resistencia a la flexión. Si bien existen varias normas IRAM sobre esta propiedad mecánica de materiales cerámicos, y la forma de evaluarla, en ellas no se mencionan valores de referencia. Por ello, se relacionan los valores obtenidos en este trabajo con la norma ASTM C410-60 (ladrillos para piso industrial), donde el valor mínimo establecido para el MOR es de 5,2 MPa. En este caso, las muestras con carozos de durazno hasta un 10\% se ajustan a la norma mencionada.

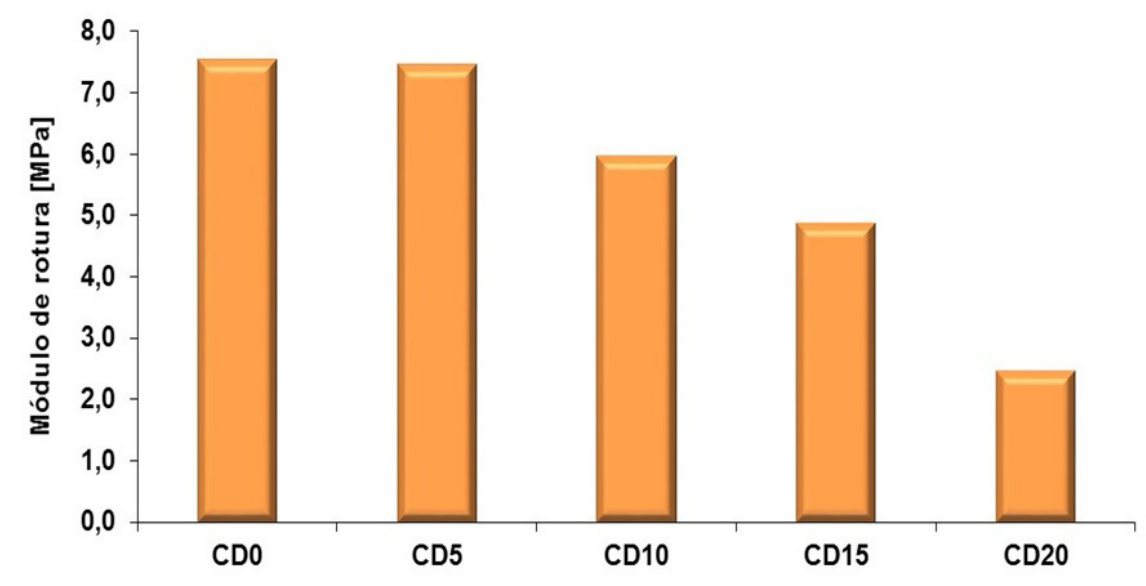

Figura 11: Resistencia a la flexión (MOR). 


\section{Conclusiones}

A partir de los resultados obtenidos, es posible concluir que es factible utilizar este material residual, carozos de durazno, como formador de poros en materiales cerámicos, ya que presenta un amplio rango de temperaturas de combustión y un porcentaje muy pequeño de cenizas que quedan incorporadas en el ladrillo, lo que hace que la probabilidad de formación de grietas durante la cocción resulte baja.

Los productos obtenidos resultaron con coloraciones homogéneas, sin desgranamiento de estructura en bordes y esquinas, lo que indica un adecuado grado de sinterización. Es importante mencionar que las temperaturas de sinterización que se utilizaron son las mismas que las utilizadas en la industria cerámica para arcillas sin agregados.

Las piezas cerámicas producidas presentan buenas propiedades en todos los contenidos ensayados. Las propiedades mecánicas de las mismas se ajustan a los requerimientos del mercado (considerando la norma ASTM C410-60), sólo para agregados de hasta 10\% en volumen de descarte. Para contenidos superiores se obtienen propiedades mecánicas inferiores a las requeridas.

Esto conduce a la posibilidad de utilizar este residuo biomásico con el objetivo de aumentar la porosidad en cerámicos, reemplazando los compuestos orgánicos derivados del petróleo habitualmente usados industrialmente, a la vez que se le da mayor utilidad a este residuo agroindustrial.

\section{Agradecimientos}

Los autores desean agradecer a la Comisión de Investigaciones Científicas de la Provincia de Buenos Aires - CIC, y a la Universidad Tecnológica Nacional, por el apoyo económico otorgado para realizar esta investigación. 


\section{Referencias}

Alvarez Torrellas S., García Lovera R., Escalona N., Sepúlveda C., García J. (2015). "Chemical-activated carbons from peach stones for the adsorption of emerging contaminants in aqueous solutions”. Chemical Engineering Journal, 279, 788-798.

Arroyo Gómez J., Villaroel Rocha D., Freitas Araújo K., Martínez Huitle C., Sapag K. (2018). "Applicability of activated carbón obtained from peach stone as an electrochemical sensor for detecting caffeine”. Journal of Electroanalytical Chemistry, 822, 171-176.

Bouja M., Sikkema R., Jegard M., Motola V., Dellemand J. (2019). "Biomass for energy in the EU - The support framework", Energy Policy, 131, 215-228.

Damien A. (2010). La biomasa. Fundamentos, tecnologías y aplicaciones. Madrid: Ed. AMV-Mundi Prensa.

Da Silva S., Chaves Arantes M., de Andrade J., Andrade C., Prokisio T. (2020). "Influence of physical and chemical compositions on the properties and energy use of lignocellulosic biomass pellets in Brazil”, Renewable Energy, 174 [1], 1870-1879.

De Velasco Maldonado P., Hernández-Montoya V., Montes-Morán M. (2016). "Plasmasurface modification vs air oxidation on carbon obtained from peach stone: Textural and chemical changes and the efficiency as adsorbents", Applied Surface Science, 384, 143-151.

Ferrer M., Pena Rodríguez G., Dulce H. (2011). "Construcción y caracterización de cerámicas porosas a partir de espumas de poliuretano y barbotinas de arcillas rojas”, Revista Colombiana de Física, 43 [2] 446-450.

Mao G., Huang N., Chen L., Weng H. (2018). "Research on biomass energy and environment from the past to the future: A bibliometric analysis", Science of the Total Environment, 635 [1], 1081-1090.

Martin Martínez M., Alvarez Torrellas S., García J., Silva A., Gomes H. (2018) "Exploring the activity of chemical-activated carbons synthesized from peach stones as metal-free catalysts for wet peroxide oxidation”. Catalysis Today, 313, 20-25.

Nunez L., Causer T., Ciolkosz D. (2020). "Biomass for energy: A review on supply chain management models", Renewable and Sustainable Energy Reviews, 120, Art. 109658.

Quaranta N., Unsen M., López H., Cristóbal A. (2017). "Characterization of peach stones and analysis of their use as pore former in ceramic materials", Journal of Fundamentals of Renewable Energy and Applications, 7[9], 49.

Rabaçal M., Fernandes U., Costa M. (2013) "Combustion and emission characteristics of a domestic boiler fired with pellets of pine, industrial wood wastes and peach stones", Renewable Energy, 51, 220-226.

Saidur R., Abdelaziz E., Demirbas A., Hossain M., Mekhilef S. (2011). "A review on biomass as fuel for boilers", Renewable and Sustainable Energy Reviews, 15 [5], 2262-2289.

Tsoncheva T., Mileva A., Tsyntsarski B., Paneva D., Petrov N. (2018). "Activated carbon from Bulgarian peach stones as a support of catalysts for methanol decomposition”. Biomass and Bioenergy, 109, 135-146. 
Vázquez Villanueva R., Marina M., García M. (2015). "Revalorization of a peach (Prunus persica L. Batsch) byproduct: Extration and characterization of ACE-inhibitory peptides from peach stones". Journal of Functional Foods, 18 A, 137-146. 